\title{
Strategic and perceptual factors producing tilt contrast in dot localization
}

\author{
DAVID J. BRYANT and ILAVENIL SUBBIAH \\ Northeastern University, Boston, Massachusetts
}

\begin{abstract}
Encoding spatial location in a frame of reference is often biased by both perceptual and strategic factors. For example, tilt contrast occurs when a line presented in the frame of horizontal and vertical axes appears to be repulsed from the nearest axis, including the diagonal axis of symmetry, due to symmetry perception mechanisms. Research has demonstrated, however, that people can adopt particular viewing strategies that eliminate this effect. In Experiment 1, a similar tilt contrast effect was observed when subjects reproduced from memory the position of a single dot in this reference frame. It was hypothesized that this effect resulted from a combination of strategic and perceptual factors. Specifically, people employ an origin strategy, coding the location of the dot relative to the origin of the horizontal and vertical axes, thereby establishing a virtual line that appears tilted away from the axes due to the same perceptual processes affecting physically present lines. Two additional experiments support this hypothesis. In Experiment 2, no clear tilt contrast effect was observed in a perception condition, indicating that the tilt effect for dots cannot be accounted for by purely perceptual processes. In Experiment 3, the tilt contrast effect was found to be contingent upon the use of the origin strategy as opposed to a different strategy. The results demonstrate the importance of a viewer's strategy in determining the pattern of distortion observed in spatial encoding.
\end{abstract}

The encoding of spatial location depends on the use of a frame of reference. In a pictorial figure, a frame of reference can be composed of any salient or familiar features to which other points or regions are related. Some examples include dots or point-like landmarks (Coren \& Girgus, 1980), enclosing figures (Huttenlocher, Hedges, \& Duncan, 1991; Nelson \& Chaiklin, 1980), horizontal and vertical axes (Schiano \& Tversky, 1992; Tversky \& Schiano, 1989), the boundaries of the display (Hartley, 1978; Lederman \& Taylor, 1969), and grids or distance markers (Bryant \& Subbiah, in press). Using a reference frame invokes both perceptual, or bottom-up, processes and strategic, or top-down, processes. This is especially true for figures that have a symbolic meaning, such as graphs or maps. Both kinds of processes, however, can induce some kind of systematic bias in the encoding of location.

Many perceptual factors have been found to bias the encoding of location. One example is the attraction of points toward landmarks or reference points (Sadalla, Burroughs, \& Staplin, 1980). Thus, dots in a visual display

This research was presented at the 33rd Annual Meeting of the Psychonomic Society in St. Louis. Our thanks go to Margaret Jean IntonsPeterson, Adam Reeves, Barbara Tversky, Peter Wenderoth, and two anonymous reviewers for their helpful suggestions, to Rhea Eskew for his expert statistical advice, and to Tova Sapoznik for her help with collecting and coding subjects' responses. Correspondence regarding this article should be sent to D. Bryant, Department of Psychology, 125 Nightingale Hall, Northeastern University, Boston, MA 02115 (e-mail: bryant@northeastern.edu). are typically misjudged as being closer to a reference dot than they really are (Coren \& Girgus, 1980). The positions of dots are also misperceived relative to the enclosing reference frames. Lederman and Taylor (1969) found that visual and tactile perception of the location of a point on a rectangular card is distorted toward the edge of the card. Similarly, Hartley (1978) found that dots presented inside a square are misperceived closer to the boundary of the square. The perceptual mechanisms that produce such attraction effects are not well understood, but Coren and Girgus (1980) suggest that they are related to Gestalt principles of organization, notably, grouping by proximity.

The perception of lines is also biased by reference frames, as can be seen in the phenomenon of tilt contrast or acute-angle expansion (e.g., Blakemore, Carpenter, \& Georgeson, 1970; Bouma \& Andriessen, 1970; Carpenter \& Blakemore, 1973; Wenderoth, Parkinson, \& White, 1979). Tilt contrast occurs when subjects judge the orientation of a test line relative to one or more inducing lines. In general, lines converging at an acute angle appear to be separated by a greater angle than they really are.

Schiano and Tversky (1992; Experiments 1 and 2) have demonstrated tilt contrast with lines presented within the frame of reference created by orthogonal horizontal and vertical axes. Their subjects reproduced simple graph-like figures from memory (memory condition) or while viewing the figures (perception condition). The figures were composed of orthogonal vertical and horizontal axes and a third line that converged on the origin of the axes at 
orientations that varied $5^{\circ}$ to $85^{\circ}$ from the horizontal axis in $5^{\circ}$ steps. When the orientations of the subjects' reproductions were compared with the stimulus orientation, a complex cyclical pattern of errors emerged in both the perception and memory conditions (the present Figure 6A displays a very similar pattern). The angular errors were positive at small angles, became negative as the stimulus angle neared $45^{\circ}$, became positive at angles immediately greater than $45^{\circ}$, and finally became negative at stimulus angles near $90^{\circ}$. This pattern of errors indicates that the target lines appeared tilted away from both the horizontal and vertical axes as well as the diagonal $45^{\circ}$ line (which was not drawn in the stimulus figures).

Schiano and Tversky (1992) suggested that the observed pattern of errors is understandable in terms of perceptual factors that bias the perception of symmetry and pattern orientation (see Wenderoth \& Johnstone, 1987). Carpenter and Blakemore (1973) have proposed that tilt contrast is the result of lateral inhibition among orientation-specific cortical cells early in visual perception, but tilt contrast occurs between test lines and "virtual" axes of symmetry, such as the diagonal in Schiano and Tversky's stimuli (Hartley, 1982; Wenderoth \& Curthoys, 1974; Wenderoth, Johnstone, \& van der Zwan, 1989). Because the inhibition of local orientation cells could not explain this finding, Wenderoth and colleagues (Beh, Wenderoth, \& Purcell, 1971; Wenderoth \& Beh, 1977; Wenderoth et al., 1989) proposed the major axes hypothesis, which states that a line will appear tilted away from the nearest axis of mirror symmetry in the reference frame, including axes not actually present in the figure. In the orthogonal frame discussed here, axes of symmetry correspond to the physical horizontal and vertical axes and the virtual diagonal formed by those axes. According to the major axes hypothesis, axes of symmetry are treated as weak but real lines by the visual system and can produce tilt contrast. Wenderoth and Johnstone suggest that this is because higher level cells in the visual cortex respond to the global pattern orientation, which makes axes of symmetry highly salient. Thus, the $45^{\circ}$ line, though not present, may activate cortical cells sensitive to that orientation, inhibiting cells responding to test lines at near orientations.

In addition to perceptual factors, conceptual or strategic factors also affect the encoding of spatial location. Nelson and Chaiklin (1980) performed a dot localization study, in which subjects viewed a dot located on a line passing through the center of a circle. The subjects tended to reproduce the dot closer to the boundary of the circle when they responded from memory, but not when they responded to a perceptually available display. Instead, in the perception condition, dots near the boundary were repulsed by it. The results of the perception condition contrast with the attraction effect observed in other studies (Hartley, 1978; Lederman \& Taylor, 1969). Nelson and Chaiklin suggested that, because the subjects were allowed unlimited time to view the stimuli in the perception con- dition, they consulted multiple reference points along the circular boundary. The multiple landmarks had additive effects and eliminated the systematic bias observed in the memory condition.

Huttenlocher, Hedges, and Duncan (1991) have argued that Nelson and Chaiklin's (1980) perception condition data reflect a hierarchical representation of location. According to their model, viewers code the location of a dot inside a circle at two levels-a categorical level and a finegrain metric level specifying the dot's polar coordinates in the circle. Viewers divide the circle into four regions or categories by imposing horizontal and vertical axes with their origin at the center of the circle. Each region is represented by a prototypical position at or near the center of the region. Assuming that coding of fine-grain location is imprecise but unbiased, viewers use categorical information during reproduction of the dot to enhance their accuracy. In four experiments that partially replicated Nelson and Chaiklin's paradigm, Huttenlocher et al. found that subjects tended to misplace dots closer to the prototypical center of the region in which the dot had appeared.

Encoding of lines is also affected by viewer strategy. Schiano and Tversky (1992) found that the tilt contrast effect did not occur when subjects were instructed to judge the orientation of a line with respect to the imaginary $45^{\circ}$ line formed by horizontal and vertical axes rather than with respect to the axes themselves. Instead, the lines at all stimulus angles from $5^{\circ}$ to $85^{\circ}$ appeared to be attracted toward the diagonal. This pattern is also consistent with repulsion from the horizontal and vertical axes, but does not explain why the lines were not repulsed by the diagonal axis of mirror symmetry, as predicted by Wenderoth's major axes hypothesis (e.g., Wenderoth et al., 1989), and observed in Schiano and Tversky's first two experiments. It is unclear why instructions to use the diagonal as a referent produces an attraction effect toward the diagonal, given that tilt contrast occurs between test lines and virtual axes of symmetry (Wenderoth \& Beh, 1977; Wenderoth et al., 1989). Schiano and Tversky argue that the attraction to the diagonal is entirely a strategic effect, similar to cognitive landmark effects observed in more abstract distance estimation tasks (e.g., Rosch, 1975). However, they do not offer a detailed account of what kinds of cognitive heuristics the viewers might have employed to encode lines relative to the diagonal, or how this strategy overcame tilt contrast. Nevertheless, Schiano and Tversky's results demonstrate that viewers can adopt different frames of reference within a given figure.

Which features are used as a frame of reference depends on how a pictorial figure is interpreted. Tversky and Schiano (1989) found that subjects who were told that a line in a pair of horizontal and vertical axes (with tick marks) represented a graph tended to reproduce the line closer to the diagonal. This suggests that viewers spontaneously adopt the $45^{\circ}$ line as a cognitive landmark for encoding graphs. Subjects who were told that the same 
figures represented maps, however, displayed no attraction toward the diagonal, indicating that they did not use the $45^{\circ}$ line as landmark for maps.

Although strategic factors are clearly important in encoding location, disentangling their effects from perceptual factors can be difficult because the exact nature of the viewer's strategy is unknown. People are not passive observers; they bring a great deal of conceptual knowledge to bear when interpreting a pictorial figure, and actively use elements of the figure to create frames of reference. For example, Bryant and Subbiah (in press) found that subjects created subjective landmarks in a visual display when encoding the position of a dot in a square that had distance markers along its sides. The subjects tended to reproduce dots closer to the imaginary intersection points of these distance markers, suggesting that they mentally projected from the distance markers and used the intersections as landmarks.

The present experiments were designed to explore in detail the effect of a viewer's encoding strategy on one particular kind of reference frame-that formed by orthogonal vertical and horizontal axes. We chose this reference frame for several reasons. First, it is relatively simple, and we were able to devise two intuitive but detailed encoding strategies for dot localization. Whereas most previous research has focused on the impact of selecting one reference frame or another (e.g., Tversky \& Schiano, 1989), we hoped to determine how particular strategies for using a reference frame would bias encoding. A second advantage was the availability of data on perceptual and strategic biases in line and dot encoding within orthogonal axes (Hartley, 1978; Schiano \& Tversky, 1992; Wenderoth, 1983), which provided the basis for predicting the pattern of bias produced by each strategy. Finally, the figures used in these experiments are similar to simple graphs or maps. Learning about the strategies that people employ to code spatial information in this frame will help us to predict errors in memory and perception of more meaningful and complex stimuli.

The goal of this study was to demonstrate that encoding of location within a frame of reference depends on the use of some cognitive heuristic or strategy. The strategy need not be a conscious plan on the part of the viewer, but is nonetheless a definite set of procedures that code some measurement of location with respect to the reference frame. For any reference frame, there should be multiple encoding strategies available to the observer. Each of these would produce a different pattern of distortion in spatial encoding as a result of the distinct conceptual and perceptual mechanisms invoked. The latter point has been demonstrated in studies that have contrasted the effects of different tasks or conceptualizations of pictorial figures (Schiano \& Tversky, 1992; Tversky \& Schiano, 1989; Wenderoth, 1983), but the present study attempts to link distortion in localization to specific encoding strategies.

To study this issue, we combined the dot localization task (e.g., Bryant \& Subbiah, in press; Huttenlocher et al., 1991) with the orientation paradigm employed by Schiano and Tversky (1992). Dot localization has previously been used to study the processes underlying orientation encoding, and two studies suggest that encoding of dot location can be distorted by orthogonal axes in a fashion similar to line orientation. Hartley (1982) had subjects align a dot presented in the frame of two orthogonal axes with their origin point. The subjects' responses were tilted with respect to the diagonal axis of symmetry of the frame, indicating that the virtual or imaginary line from dot to origin activated the same symmetry extraction mechanisms as real lines. Wenderoth (1983) replicated this result when subjects judged the orientation of the virtual line, but found that tilt contrast did not occur when they were instructed to judge the dot's position in the frame rather than its orientation. The different tasks presumably induced different viewing strategies, which produced different results. Together, these studies suggest that localization of a dot inside horizontal and vertical axes may be subject to tilt contrast, depending on the encoding strategy the viewer adopts.

\section{EXPERIMENT 1}

In the first experiment, subjects viewed simple graphlike figures composed of two orthogonal axes and a small dot (see Figure 1). After viewing each figure, the subjects attempted to reproduce from memory the location of the dot in a set of response axes. The dots were placed on a line from the origin of the axes (this line was not actually presented in the figure); the orientation of this line was varied from $5^{\circ}$ to $85^{\circ}$ in $5^{\circ}$ increments.

We expected that strategic factors would be important in this paradigm because there are several ways in which the axes could be used to encode the location of a dot.

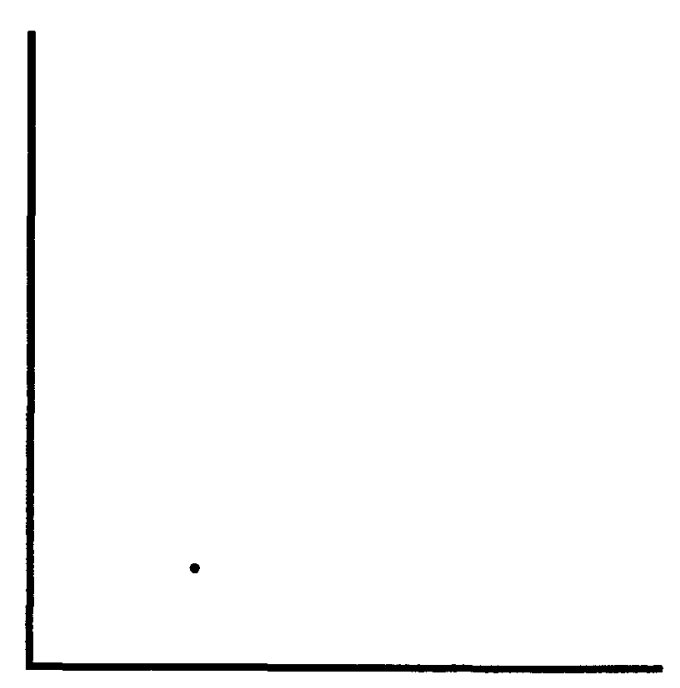

Figure 1. A sample stimulus figure used in Experiment 1 (short condition). The figures used in Experiments 2 and 3 were similar. 
One possible strategy, which we term the origin strategy, is to judge the location of the dot relative to the origin of the axes, as though there were a physical line from the origin to the dot. This would require the subject to code two pieces of information-the distance of the point from the origin and the orientation of the implied or virtual line (from origin to dot) relative to the nearest axis. This strategy is consistent with Huttenlocher et al.'s (1991) finding that people, at least sometimes, use polar coordinate systems to code the location of points in a visual display. Using this strategy makes the dot localization task functionally equivalent to Schiano and Tversky's (1992) line orientation task, except that the line in the frame would be virtual or subjective rather than physically present in the figure. If virtual lines activate symmetry perception mechanisms in the same way that real lines do (Hartley, 1982; Wenderoth, 1983), we would expect a pattern of distortion in dot localization similar to that in line reproduction, and subjects' responses should be "tilted" away from both the horizontal and vertical axes, as well as from the diagonal axis of symmetry. The ideal pattern of angular errors in dot reproduction, if errors were measured at all angles, would resemble a sine-wave function with two cycles per $90^{\circ}$. The positive peaks would be at $11.25^{\circ}$ and $56.25^{\circ}$, the negative peaks at $33.75^{\circ}$ and $78.75^{\circ}$, and the zero error crossings at $22.5^{\circ}$, $45^{\circ}$, and $67.5^{\circ}$. Note that these predictions are based on the assumption that equal repulsion is produced by the implicit $45^{\circ}$ line as the explicit horizontal and vertical axes. This assumption is consistent with Schiano and Tversky's findings with line reproduction. In the present experiments, observations were made only at $5^{\circ}$ intervals and the positive peaks should be at or near $10^{\circ}$ and $55^{\circ}$, the negative peaks at or near $35^{\circ}$ and $75^{\circ}$, and the zero crossings at or near $20^{\circ}-25^{\circ}, 45^{\circ}$, and $65^{\circ}-70^{\circ}$. Figure 2 presents the ideal predicted pattern of errors as

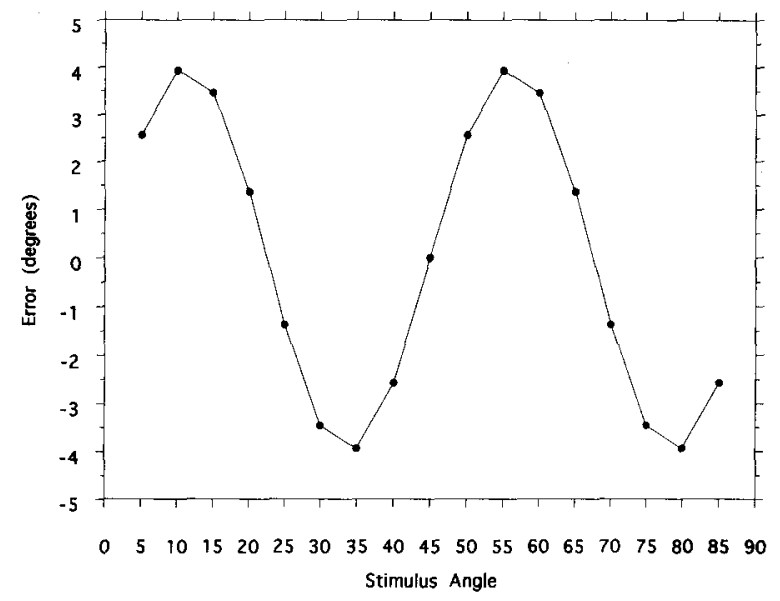

Figure 2. Ideal pattern of angle errors predicted by the origin strategy at the levels of stimulus angles used in the experiments. This figure was generated by selecting 17 data points from a sine-wave function of two cycles per $90^{\circ}$. The magnitudes of peak errors in the figure are arbitrary; the origin strategy does not predict the extent of peak error.

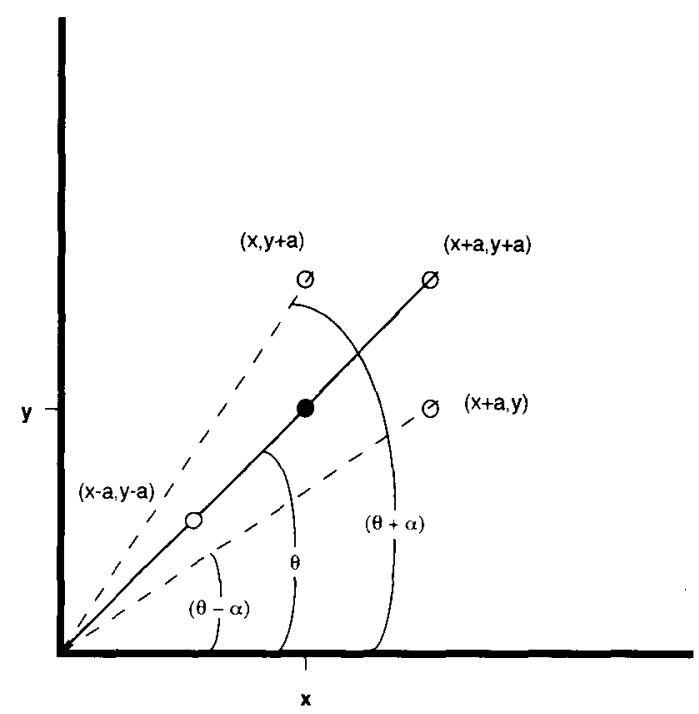

Figure 3. The geometric effect of distorting the stimulus dot's (shown as the solid dot at coordinates $x, y$ ) position along the horizontal and vertical axes. Adding or subtracting a constant, a, to both axes does not change the stimulus angle, $\theta$, but does alter the distance from origin to dot. Adding a constant, a, to the horizontal axis alone reduces the stimulus angle by the factor $\alpha$. Adding a constant, $a$, to the vertical axis alone increases the stimulus angle by the factor $\alpha$. The effects of subtracting constants from the horizontal and vertical axes can be calculated in a similar fashion.

a function of the stimulus angles employed in the present experiments.

Although subjects might adopt polar coordinates, the orthogonal axes themselves conform to axes in a Cartesian space. Therefore, another possible strategy would be to use the axes to define the Cartesian coordinates of the dot, much like using $x$ and $y$ coordinates of a graph. This could be done by estimating the dot's position along the horizontal and vertical axes separately, then projecting from these positions to an intersection in the response axes. We term this the axis strategy.

If subjects code a dot's location as two distances along the two axes, there is no reason to predict tilt contrast in dot localization, because they are not coding orientation. They could, however, systematically over- or underestimate a dot's position along either the horizontal or vertical axes, or both, and this would distort their angular responses (see Figure 3). How angular responses would be distorted depends on the nature of the bias in estimating horizontal and vertical position. Figure 4 displays three possible patterns of errors consistent with the axis strategy. If the subjects exhibit no systematic bias in estimating horizontal and vertical position, or exhibit equal over- or underestimation of position along the horizontal and vertical axes, angle errors will be zero at all stimulus angles (see also Figure 3). The subjects could exhibit unequal bias in judging position along the two axes. If overestimation along the horizontal is greater than bias on the vertical (or underestimation along the vertical is greater than underestimation along the horizontal), they would 


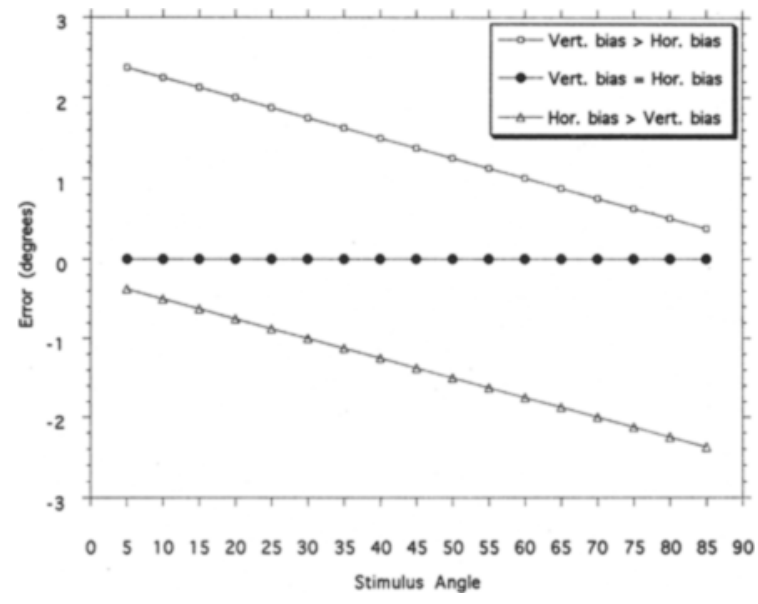

Figure 4. Three possible ideal patterns of errors predicted by the axis strategy, assuming that bias in estimating horizontal and vertical position of dots does not vary with stimulus angle. Bias refers to both over- and underestimation along an axis. The magnitudes of the positive and negative error functions in the figure are arbitrary; the axis strategy does not predict the degree of over- or underestimation along the horizontal and vertical axes.

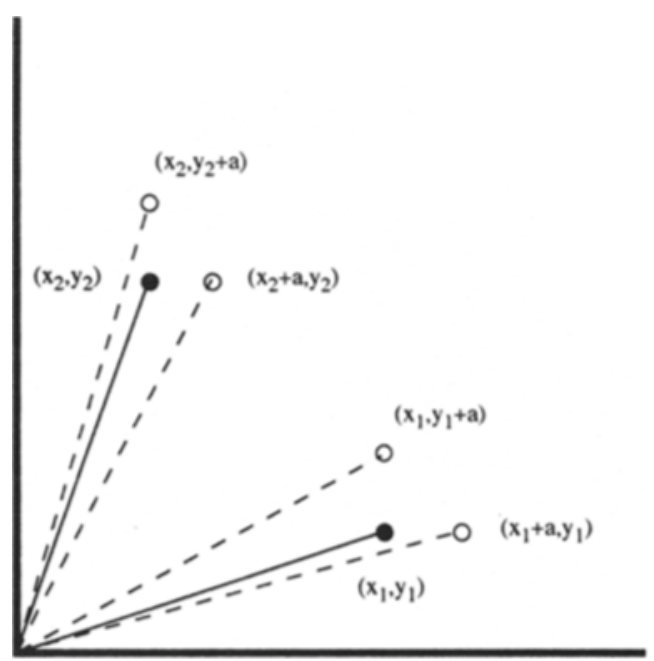

Figure 5. Adding a constant, a, to the horizontal axis produces a smaller angular underestimation at small stimulus angles than at larger stimulus angles $\left[\left(x_{1}+a, y_{1}\right)\right.$ vs. $\left.\left(x_{2}+a, y_{2}\right)\right]$. Adding a constant to the vertical axis produces a larger angular overestimation at smaller stimulus angles than at larger stimulus angles $\left[\left(x_{1}, y_{1}+a\right)\right.$ vs. $\left.\left(x_{2}, y_{2}+a\right)\right]$. The effects of subtracting constants from the horizontal and vertical axes can be determined in a similar fashion.

consistently underestimate stimulus angle. The magnitude of the underestimation, however, would be less at smaller stimulus angles than at larger stimulus angles (see Figure 5). If overestimation along the vertical is greater than overestimation along the horizontal (or underestimation along the horizontal is greater than underestimation along the vertical), they would consistently overestimate stimulus angle. The magnitude of the overestimation would be greater at smaller stimulus angles than at larger stim- ulus angles (see Figure 5). In both cases, angular error would be a linear decreasing function of the inverse tangent of perceived vertical extent divided by perceived horizontal extent and stimulus angle $\left[\Delta \theta=\tan ^{-1}(y+a / x+b)\right.$ $-\theta]$, as shown in Figure 4. Note that bias in coding position along the two axes can produce a cyclical pattern of angle errors only if the magnitude and direction of horizontal and vertical bias varies as a function of stimulus angle. ${ }^{1}$

Method
Subjects
Forty Northeastern University undergraduates participated in ex-
change for credit in an introductory psychology class.

\section{Materials}

The stimuli were simple graph-like figures, consisting of two orthogonal axes and a single point (see Figure 1). Each axis was 5 in. in length and approximately $1 / 8$ in. thick. The point was a filled circle with a diameter of $1 / 8$ in. Points were placed in the figures at angles (relative to the origin of the axes) of $5^{\circ}$ to $85^{\circ}$ in $5^{\circ}$ steps, and at a radial distance of 1-2 in. from the origin ("short" condition) and 3-4 in. ("long" condition), for a total of 34 figures. The figures were organized into booklets in a random order. A blank sheet of colored paper was placed between each stimulus to prevent the next item from showing through. Response sheets displayed the two axes without the stimulus dot. The response booklets consisted of 34 response sheets.

\section{Procedure}

Each subject received a stimulus booklet and a response booklet. They were instructed to place the stimulus booklet on the left side of the table that was in front of them and the response booklet on the right. They were told to study each stimulus for $7 \mathrm{sec}$, then turn the stimulus sheet over when instructed to do so by the experimenter. After a $7 \mathrm{sec}$ retention interval, the subjects were asked to indicate the location of the stimulus point by drawing a point on the response sheet. They were not permitted to erase or correct a response once it had been made. After making a response, the subjects turned the response sheet over and uncovered the next stimulus item. The procedure was repeated for all 34 items. The subjects were run in groups of up to 5 .

\section{Design}

The independent variables were stimulus angle $\left(5^{\circ}-85^{\circ}\right.$, in $5^{\circ}$ increments) and stimulus distance from the origin (short or long). The dependent variables were response angle error, defined as the angle of the subject's response relative to the origin and horizontal axis minus the true angle of the stimulus point, and response distance error, defined as the distance of the subject's response from the origin minus the true stimulus distance.

\section{Results}

Using a ruler and protractor, a research assistant coded the responses by drawing a straight line between the response graph's origin and the center of a subject's response point, then measuring the angle between the line and the horizontal axis (to the nearest half-degree) and the distance from the origin to the center of the response dot (to the nearest millimeter for maximum accuracy). The stimulus angle was subtracted from the response angle for each item to derive an error score. The true distance between the origin and stimulus point was likewise subtracted from the response distance to derive a distance 
error. Outliers, defined as a data point greater than a cell mean plus or minus two standard deviations, accounted for $5.5 \%$ of the angle error data in the short condition and $5.0 \%$ of the angle error data in the long condition. These scores were replaced with the cell mean for analysis.

\section{Angle Errors}

Figures $6 \mathrm{~A}$ and $6 \mathrm{~B}$ present the mean angle errors for each stimulus angle in the short and long conditions, respectively. In both cases, the function is cyclical, starting with a positive error, decreasing to negative error, increasing again, then decreasing. A repeated measures analysis of variance (ANOVA) revealed a significant main effect of stimulus angle $[F(16,304)=17.35, p<.01]$, no significant effect of stimulus distance $[F(1,19)=2.03$, $p>.05]$, but a significant interaction between stimulus
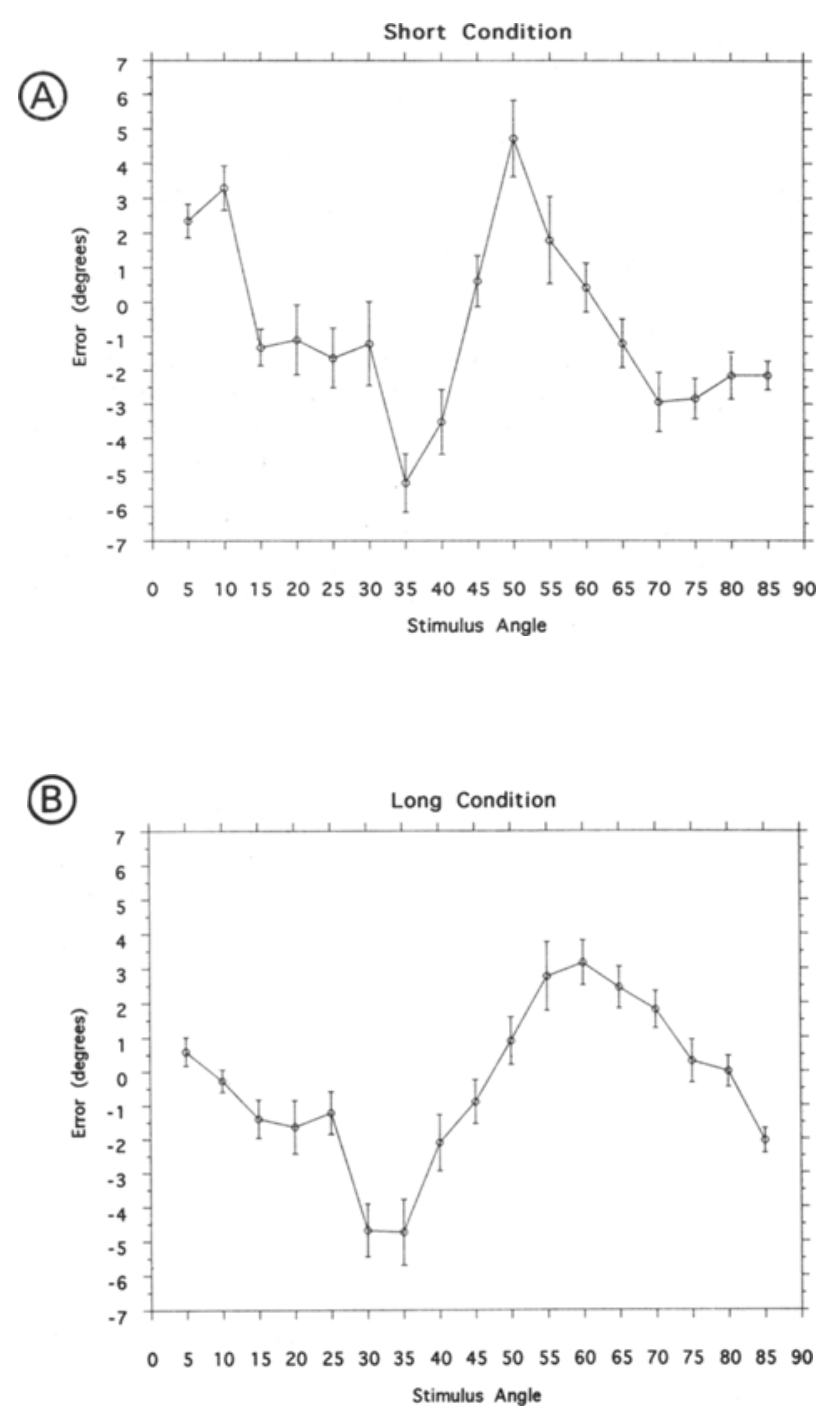

Figure 6. Mean error (in degrees) of remembered orientation as a function of stimulus angle for the short condition (A) and the long condition (B) in Experiment 1 . The vertical bars at each point represent the standard error of the means. angle and stimulus distance $[F(16,304)=7.21, p<.01]$. The angle data are discussed separately below for the short and long conditions.

Short condition. Peak overestimation occurred at $10^{\circ}$ and $50^{\circ}$, and peak underestimation at $35^{\circ}$ and $70^{\circ}$. Errors were significantly different from zero at stimulus angles of $5^{\circ}[t(19)=4.92, p<.01] ; 10^{\circ}[t(19)=6.14$, $p<.01] ; 35^{\circ}[t(19)=-6.95, p<.01] ; 40^{\circ}[t(19)=$ $-3.89, p<.01] ; 50^{\circ}[t(19)=4.75, p<.01] ; 55^{\circ}$ $[t(19)=2.78, p<.05] ; 70^{\circ}[t(19)=-3.76, p<.01]$ $75^{\circ}[t(19)=-5.00, p<.01] ; 80^{\circ}[t(19)=-3.28, p<$ $.01]$; and $85^{\circ}[t(19)=-5.62, p<.01]$. Errors at all other stimulus angles did not reliably differ from zero. A trend analysis revealed that the largest effect was fifth order, accounting for $21.53 \%$ of the total sum of squares (SS) of the main effect of stimulus angle $[F(1,19)=$ $31.86, p<.011$, which reflects the fact that there is a positive peak at $10^{\circ}$ and a negative peak at $75^{\circ}$. Trend analyses also revealed significant linear $[7.98 \%$ of SS, $F(1,19)=11.81, p<.01]$, cubic $[20.77 \%$ of SS, $F(1,19)$ $=30.74, p<.01]$, seventh order $[9.45 \%$ of SS, $F(1,19)$ $=13.99, p<.05]$, and ninth order $[10.99 \%$ of SS, $F(1,19)=16.26, p<.05]$ effects.

Long condition. Peak overestimation occurred at $5^{\circ}$ and $60^{\circ}$, and peak underestimation at $35^{\circ}$ and $85^{\circ}$. Errors were significantly different from zero at stimulus angles of $15^{\circ}[t(19)=-2.70, p<.05] ; 20^{\circ}[t(19)=$ $-2.16, p<.05] ; 30^{\circ}[t(19)=-6.46, p<.01] ; 35^{\circ}$ $[t(19)=-5.25, p<.01] ; 40^{\circ}[t(19)=-1.36, p<$ $.05] ; 55^{\circ}[t(19)=3.30, p<.05] ; 60^{\circ}[t(19)=5.16$, $p<.05] ; 65^{\circ}[t(19)=4.49, p<.05] ; 65^{\circ}[t(19)=$ $4.49, p<.05] ; 70^{\circ}[t(19)=3.33, p<.05]$; and $85^{\circ}$ $[t(19)=-5.49, p<.05]$. Errors at all other stimulus angles did not reliably differ from zero. A trend analysis revealed that the largest effect was cubic [53.77\% of SS, $F(1,19)=73.42, p<.01]$, which reflects the absence of positive and negative peaks at $10^{\circ}-15^{\circ}$ and $70^{\circ}-75^{\circ}$, respectively. Trend analyses revealed additional significant linear $[13.09 \%$ of SS, $F(1,19)=17.88, p<.01]$, fifth order [14.19\% of SS, $F(1,19)=19.37, p<.01]$, and seventh order $[9.09 \%$ of SS, $F(1,19)=12.41, p<$ .01] effects.

\section{Comparison of Short and Long Conditions With Predictions of the Origin Strategy}

The patterns of errors in the short and long conditions are similar in certain respects; they share a general cyclical pattern, with the initially positive errors becoming negative at stimulus angles approaching $45^{\circ}$ and positive errors at angles above $45^{\circ}$ becoming negative as stimulus angles approach $90^{\circ}$. However, the patterns differ in two important respects. The peak overestimation at $5^{\circ}$ in the long condition was not significantly greater than zero, and only a cubic trend was observed in the data of that condition. Thus, it was necessary to compare the patterns of errors of individual subjects against predictions of the origin strategy to determine whether the data support the conclusion that the subjects employed the origin strategy in both conditions. 
The predicted pattern of errors is a sine function of two cycles per $90^{\circ}$ (or four cycles per $180^{\circ}$ ). Such a periodic function can be analyzed by using the fast Fourier transform algorithm, or FFT (Cooley, Lewis, \& Welch, 1977). The output of this procedure is a description of the magnitude and phase of the frequency components (harmonics) present in the data. ${ }^{2}$ The magnitude of a frequency component $(\mathrm{FC})$ corresponds to the amplitude of a sinusoid of that frequency that best fits the data; frequencies of one, two, three, and four cycles per $180^{\circ}$ were tested here. For a set of data, any FC described by the FFT may represent noise or genuine periodicity. Russell (1985) describes a test of significance, with significance tables, for FCs derived by the FFT. A variable, $g$, calculated for each FC, represents the proportion of variance in the original series of values accounted for by that particular FC. The variable $g$ is calculated for a particular frequency component by dividing the square of the magnitude of the frequency component by the sum of the squares of all frequency components. In the model sine wave (four cycles per $180^{\circ}$ ), the fourth $\mathrm{FC}$ is significant, whereas all lower FCs are not. ${ }^{3}$ If the individual subjects' data conform to predictions of the origin strategy, Fourier analysis of their data should reveal a significant fourth FC, but no significant lower FCs.

The individual subjects' data were analyzed by FFT. The FFT algorithm can only be applied to data sets of $2^{n}$ data points. For this reason, the subjects' 17 data points could not be directly analyzed by FFT to determine FCs per $90^{\circ}$. It is, however, permissible to pad data sets with zeros at both ends to create a data set of $2^{n}$ items. In this case, seven zeros were padded at the beginning and eight zeroes at the end of each subject's 17 data points to create a set of 32 data points. This does not affect the magnitude of frequency components, but describes frequency components per $180^{\circ}$.

In the short condition, 14 of 20 subjects displayed a significant fourth FC, whereas only 2 subjects exhibited a significant second $\mathrm{FC}$ (at two cycles per $180^{\circ}$ ), and 2 subjects a significant third FC (at three cycles per $180^{\circ}$ ). Note that the sum of significant FCs may be less than or greater than the total number of subjects, because some subjects may have exhibited either no significant FCs or multiple significant FCs. In the long condition, 12 of 20 subjects exhibited a significant fourth FC, whereas only 2 subjects exhibited a significant first $\mathrm{FC}, 4$ subjects a significant second FC, and 2 subjects a significant third FC. Thus, in both conditions, the majority of the subjects produced patterns of errors conforming to the sine function pattern predicted by the origin strategy. Few subjects produced error patterns containing periodicities consistent with other functions.

\section{Distance Errors}

A repeated measures ANOVA revealed a significant effect of stimulus angle on distance errors $[F(16,304)=$ $3.44, p<.01]$, but neither the main effect of stimulus distance $[F(1,19)=1.97, p>.05]$ nor the interaction of stimulus angle and stimulus distance $[F(16,304)=$ $1.49, p>.05$ ] was significant. The subjects displayed a slight tendency to overestimate the distance between the graph's origin and the stimulus point, but the absolute error was relatively small and the distance error did not display any clear pattern. In the short condition, the mean distance error reliably differed from zero only at stimulus angles of $25^{\circ}[t(19)=2.67, p<.05]$ and $40^{\circ}[t(19)$ $=3.88, p<.01]$. In the long condition, mean error reliably differed from zero only at stimulus angles of $5^{\circ}[t(19)$ $=3.40, p<.05] ; 10^{\circ}[t(19)=2.40, p<.05] ; 15^{\circ}$ $[t(19)=3.80, p<.01]$; and $75^{\circ}[t(19)=3.21, p<$ $.05]$.

\section{Discussion}

Memory for dot location was biased away from the two orthogonal axes and the $45^{\circ}$ line, replicating the tilt contrast effect observed by Schiano and Tversky (1992; Experiments 1 and 2) with line reproduction. The subjects' responses, however, were not simply repulsed by each axis separately, nor did the subjects simply over- or underestimate the position along the horizontal and vertical axes (as might be true if they used the axis strategy). Consistent with this idea is that origin-to-dot distance showed no systematic bias, and error was controlled by the orientation of dots relative to the axes. Note that the origin-todot distance was not varied systematically, which precludes making any general conclusion about people's tendency to over- or underestimate the radial distance of dots in graph-like figures.

This pattern of distortion suggests that subjects employed the origin strategy when encoding figures. According to this strategy, the subjects judged the location of the dot relative to the origin of the axes, implying a virtual straight line from the origin to the dot (the virtual line need not be explicitly imagined). As Hartley (1982) has demonstrated, virtual lines appear tilted away from the axes of symmetry. Thus, when a subject reproduces the dot on the virtual line, the response falls at an angle that is tilted away from the nearest axis of symmetry.

Two additional experiments were performed to test this account. In Experiment 2, subjects reproduced dot location in a perception condition. It is necessary to compare memory and perception of figures to determine whether the effects observed in Experiment 1 are purely perceptual in nature, or whether they reflect strategic factors. Tversky and Schiano (1989) have argued that perceptual processes should produce similar patterns and magnitudes of distortion in perception and memory conditions, because errors in memory would reflect only distortions that arose during perception. Strategic factors, on the other hand, often have a larger effect in memory than perception because veridical and metric information about the display are unavailable (see, e.g., Bransford \& Johnson, 1972; Cermak \& Craik, 1979). To the extent that tilt contrast for dots reflects the use of the origin strategy, the effect should be weaker or absent in the perception condition. In Experiment 3, subjects were instructed to adopt 
either the origin or axis strategy to test the hypothesis that the tilt contrast effect depends on the use of the origin strategy. If this is the case, a different pattern of errors should emerge when they employ the axis strategy, which does not involve encoding the orientation of the dot relative to the origin point.

\section{EXPERIMENT 2}

\section{Method}

\section{Subjects}

Twenty Northeastern University undergraduates participated in exchange for credit in an introductory psychology class.

\section{Materials}

Given that the distance from origin to stimulus dot did not have a significant effect on angular error in Experiment 1, the short and long conditions were collapsed to a single range of stimulus distances in the present experiment. The stimuli were 17 graph-like figures similar to those used in Experiment 1. Again, each figure consisted of two orthogonal axes and a single point. Each axis was $5 \mathrm{in}$. in length and approximately $1 / 8 \mathrm{in}$. thick. The point was a filled circle, with a diameter of $1 / 8$ in. Dots were placed in the figures at angles of $5^{\circ}$ to $85^{\circ}$ from the origin, in $5^{\circ}$ steps, and at a distance of between 2 and 3 in. from the origin of the axes. The 17 figures were organized into booklets in a random order. A blank sheet of colored paper was placed between each stimulus to prevent the next item from showing through. Response booklets contained 17 response sheets consisting of the two axes with no dot.

\section{Procedure}

The procedure was similar to that of Experiment 1, except that the subjects were allowed to view the stimulus figures at their own pace and make their response while viewing the stimulus figure. No erasures or corrections were allowed. The subjects were run in groups of up to 5 .

\section{Design}

The independent variable was stimulus angle $\left(5^{\circ}-85^{\circ}\right.$, in $5^{\circ}$ increments). The dependent variables were response angle error and response distance error, both defined as in Experiment 1.

\section{Results and Discussion}

Outliers accounted for $5.5 \%$ of the angle error data and were replaced with the cell mean for analysis.

\section{Angle Errors}

Figure 7 presents mean angle error for each stimulus angle. A repeated measures ANOVA revealed a significant main effect of stimulus angle $[F(16,304)=5.68$, $p<.01]$, and the function is cyclical. However, unlike the previous results, errors at stimulus angles of $5^{\circ}$ and $10^{\circ}$ are negative rather than positive. Peak overestimation occurred at $50^{\circ}$, and peak underestimation at $25^{\circ}$ and $85^{\circ}$. Errors were significantly different from zero at stimulus angles of $5^{\circ}[t(19)=-4.36, p<.01] ; 10^{\circ}[t(19)=$ $-4.46, p<.01] ; 15^{\circ}[t(19)=-4.83, p<.01] ; 20^{\circ}$ $[t(19)=-2.68, p<.05] ; 25^{\circ}[t(19)=-3.42, p<$ $.01] ; 50^{\circ}[t(19)=2.56, p<.05] ;$ and $60^{\circ}[t(19)=2.54$, $p<.05]$. Error at $85^{\circ}$ was significantly less than zero $[t(19)=-1.82, p<.05]$ in a one-tailed test. Errors at all other stimulus angles did not reliably differ from zero. Trend analyses revealed that the largest effect was cubic

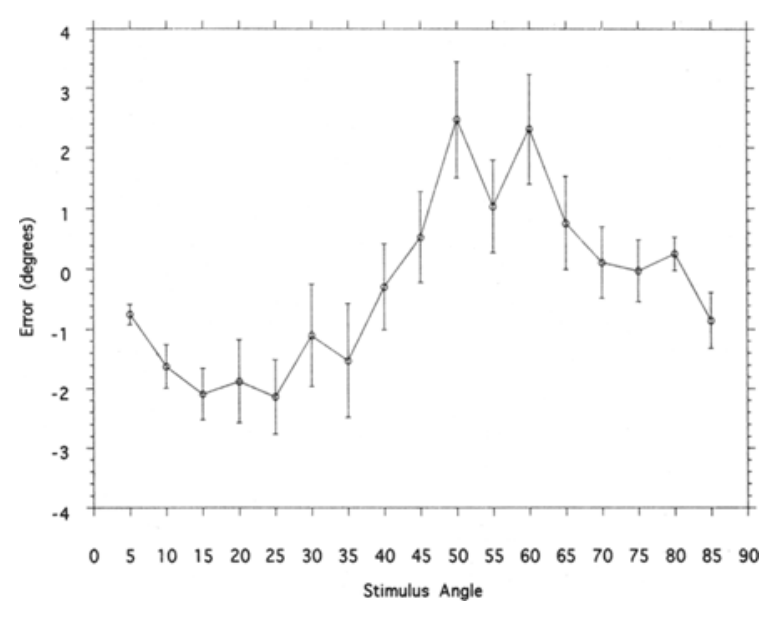

Figure 7. Mean error (in degrees) of perceived orientation as a function of stimulus angle in Experiment 2.

$[29.24 \%$ of SS, $F(1,19)=26.60, p<.01]$, which reflects the absence of a positive peak before $45^{\circ}$ and a negative peak before $85^{\circ}$. Trend analyses revealed additional significant linear $[28.85 \%$ of $S S, F(1,19)=26.23$, $p<.01]$, quadratic $[16.36 \%$ of SS, $F(1,19)=14.88$, $p<.01]$, and fourth order $[9.03 \%$ of SS, $F(1,19)=$ $8.22, p<.01]$ effects.

\section{Comparison of Error Patterns With Predictions of the Origin Strategy}

Individual subjects' data were analyzed by FFT, as in Experiment 1, and compared with the model sine function predicted by the origin strategy. Only 8 subjects exhibited a significant fourth $\mathrm{FC}$ as predicted by the model. Three subjects exhibited a significant first FC, 4 subjects a significant second FC, and 7 subjects a significant third FC. Generally, individual subjects' error patterns were not consistent with the use of the origin strategy.

\section{Distance Errors}

A repeated measures ANOVA revealed no significant effect of stimulus angle on distance errors $[F(16,304)=$ $1.43, \mathrm{n} . \mathrm{s}$.$] . The subjects did not reliably over- or under-$ estimate distance from the origin, and mean error did not differ significantly from zero at any stimulus angle.

\section{Conclusions}

The pattern of errors was generally inconsistent with predictions of the origin strategy, and mean errors were smaller than those observed in the memory condition (Experiment 1). There did seem to be some repulsion of dots from the imaginary $45^{\circ}$ line, which is consistent with tilt contrast. The absence of a strong tilt contrast effect indicates that tilt contrast for dots cannot be explained in terms of perceptual processes. Instead, a strategic factor, which has a more pronounced effect in memory than perception, mediates tilt contrast for dots. Our explanation is that the subjects used the origin strategy when encoding location to convert the dot's position into its orientation 
and origin-to-dot distance. This strategy, however, explains the tilt bias in terms of perceptual symmetry extraction mechanisms that distort the encoding of a virtual line (Hartley, 1982; Wenderoth, 1983). If it is true that perceptual and strategic factors combine to produce tilt contrast in dot localization, it should be possible to observe the effect in a perceptual condition when subjects are instructed in the use of the origin strategy. In other words, if tilt contrast in dot localization depends on the use of the origin strategy, it should be observed only when subjects employ that strategy and not when they are induced to adopt a different strategy. We tested this hypothesis in Experiment 3.

\section{EXPERIMENT 3}

In this experiment, subjects viewed and reproduced the same figures that were used in Experiment 2, but were instructed to use a particular strategy. Half of the subjects were told to use the origin strategy by imaging a line from the dot to the origin of the axes, and the other half were instructed to use the axis strategy by estimating the dot's position along each axis separately.

\section{Method}

\section{Subjects}

Forty Northeastern University undergraduates participated in exchange for credit in an introductory psychology class.

\begin{abstract}
Materials and Procedure
The materials were those used in Experiment 2. The procedure was also the same as that of Experiment 2, except that the subjects were instructed to use one of two strategies. The subjects viewed the stimulus figures at their own pace and made each response while viewing the stimulus figure. In the origin strategy condition, they were told to imagine a line from the origin of the axes to the point, then imagine that line on the response sheet as a way of reproducing the position of the dot. In the axis strategy condition, they were instructed to estimate the position of the dot along each axis separately (i.e., to determine its $x$ and $y$ locations on the axes). Then they were to make marks on the two axes of the response sheet corresponding to the dot's position and project to where the dot was located in the figure space. The subjects were run in groups of up to 5 .
\end{abstract}

\section{Design}

The independent variables were stimulus angle $\left(5^{\circ}-85^{\circ}\right.$, in $5^{\circ}$ increments), and encoding strategy (origin or axis). Encoding strategy was varied between subjects. The dependent variables were response angle error and response distance error, defined as in the previous experiments.

\section{Results and Discussion}

Outliers accounted for $4.4 \%$ of the angle error data in the origin strategy condition and $2.7 \%$ of the angle error data in the axis strategy condition and were replaced with the cell mean for analysis.

\section{Angle Errors}

Figures $8 \mathrm{~A}$ and $8 \mathrm{~B}$ present mean angle error for each stimulus angle in the origin and axis strategy conditions, respectively. A repeated measures ANOVA revealed a
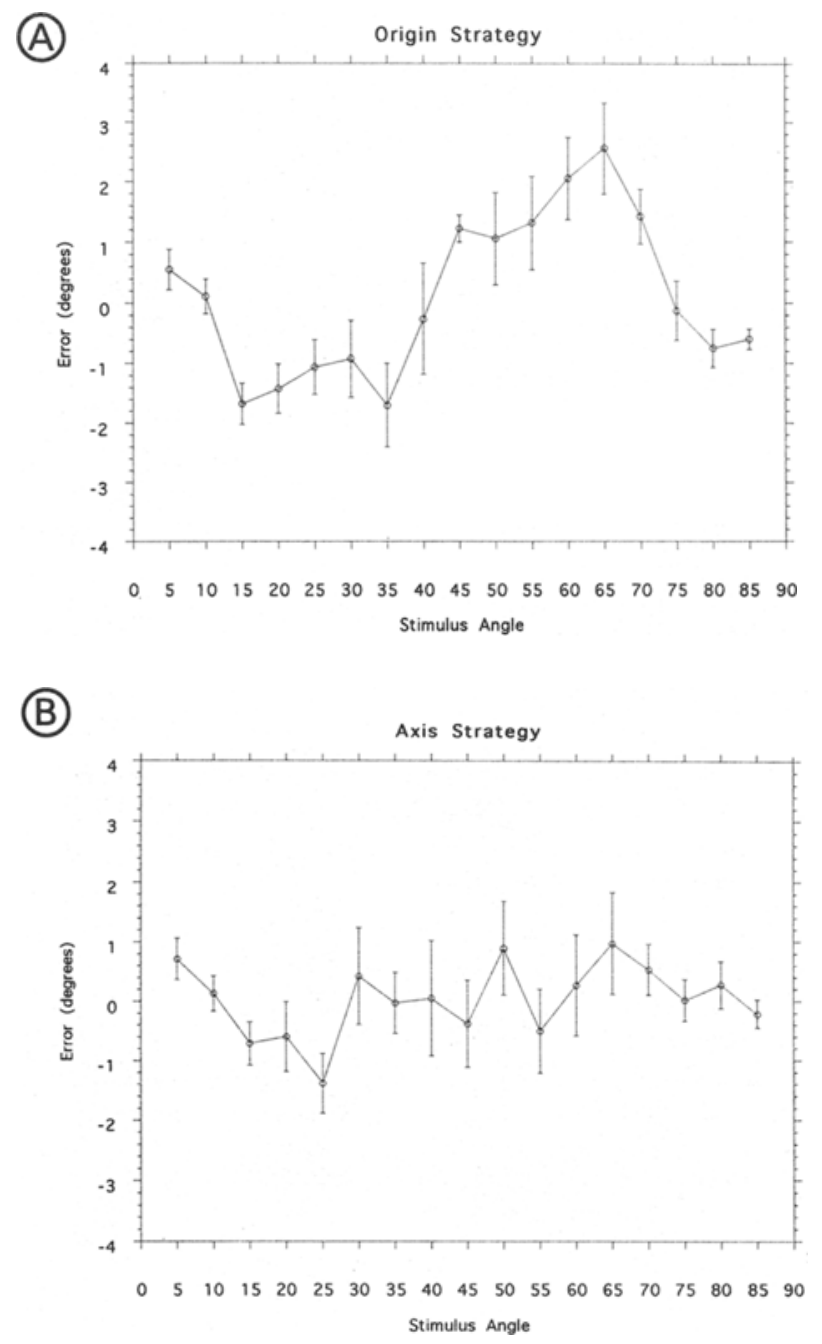

Figure 8. Mean error (in degrees) of perceived orientation as a function of stimulus angle for the origin strategy $(A)$ and the axis strategy (B) in Experiment 3.

significant main effect of stimulus angle $[F(16,304)=$ $4.81, p<.01]$, but no significant effect of encoding strategy $[F(1,19)=.008$, n.s. $]$. Thus, overall mean error in the two conditions did not differ. The interaction of stimulus angle and encoding strategy was significant $[F(16,304)=1.87, p<.05]$. Angle data are discussed separately below for the origin and axis strategy conditions.

Origin strategy condition. Peak overestimation occurred at $5^{\circ}$ and $65^{\circ}$, and peak underestimation at $35^{\circ}$ and $80^{\circ}$. Errors were significantly different from zero at stimulus angles of $5^{\circ}[t(19)=1.73, p<.05$; one-tailed test $] ; 15^{\circ}[t(19)=-4.19, p<.01] ; 20^{\circ}[t(19)=$ $-3.46, p<.01] ; 25^{\circ}[t(19)=-2.33, p<.05] ; 35^{\circ}$ $[t(19)=-2.43, p<.05] ; 60^{\circ}[t(19)=3.00, p<.01] ;$ $65^{\circ}[t(19)=3.33, p<.01] ; 70^{\circ}[t(19)=3.14, p<$ $.01] ; 80^{\circ}[t(19)=-2.37, p<.05] ;$ and $85^{\circ}[t(19)=$ $-3.52, p<.01]$. Errors at all other stimulus angles did not reliably differ from zero. Trend analyses revealed that the largest effect was cubic $[60.39 \%$ of SS, $F(1,19)=$ 
$58.92, p<.01$ ]; although there is a negative peak at $80^{\circ}$, the first positive peak is at $5^{\circ}$. Trend analyses revealed additional significant linear $[13.39 \%$ of SS, $F(1,19)=$ $13.39, p<.01]$, quadratic $[5.40 \%$ of SS, $F(1,19)=$ $5.27, p<.05]$, and fifth order $[5.74 \%$ of SS, $F(1,19)=$ $5.60, p<.05]$ effects.

Axis strategy condition. The pattern of errors was very different from that in the origin strategy condition and lacked a cyclical appearance. In fact, there appears to be no systematic bias in errors in this condition. A repeated measures ANOVA revealed no significant effect of stimulus angle in this condition $[F(16,304)=1.10$, n.s. $]$. Moreover, errors did not reliably differ from zero at any stimulus angle.

\section{Comparison of Origin and Axis Strategy Conditions With Predictions of the Origin Strategy}

Individual subjects' data were analyzed by FFT, as in Experiment 1, and compared with the model sine function predicted by the origin strategy. In the origin strategy condition, 12 of 20 subjects exhibited a significant fourth FC, whereas only 3 subjects exhibited a significant first FC, 3 subjects a significant second FC, and 4 subjects a significant third FC. The majority of the subjects' error patterns were consistent with the use of the origin strategy. In the axis strategy condition, only 6 subjects exhibited a significant fourth FC. Three subjects exhibited a significant second FC, and 6 subjects a significant third FC. The subjects' error patterns were generally inconsistent with the function predicted by the origin strategy.

\section{Distance Errors}

A repeated measures ANOVA revealed a significant effect of encoding strategy $[F(1,19)=5.90, p<.05]$, but neither the main effect of stimulus angle $[F(16,304)=$ 0.95 , n.s.] nor the interaction of stimulus angle and encoding condition $[F(16,304)=0.61$, n.s. $]$ was significant. In the origin strategy condition, the subjects had a tendency to overestimate the distance between the graph's origin and the stimulus point, but distance error did not vary cyclically with stimulus angle. In this condition, mean distance error was reliably greater than zero at stimulus angles of $5^{\circ}[t(19)=3.01, p<.01] ; 15^{\circ}[t(19)=$ $2.63, p<.05] ; 25^{\circ}[t(19)=2.15, p<.05] ; 35^{\circ}[t(19)$ $=2.38, p<.05] ; 40^{\circ}[t(19)=2.21, p<.05] ; 60^{\circ}$ $[t(19)=2.25, p<.05] ; 65^{\circ}[t(19)=2.88, p<.01]$; $70^{\circ}[t(19)=3.43, p<.01] ;$ and $80^{\circ}[t(19)=2.81, p<$ $.05]$. At all other stimulus angles, error did not differ significantly from zero. In the axis strategy condition, mean error did not differ significantly from zero at any stimulus angle.

\section{Conclusions}

The results replicate the tilt contrast effect in dot localization in a perception condition, although the magnitude of errors tended to be smaller than in the memory condition (Experiment 1). When instructed to use the origin strategy, the subjects displayed a pattern of errors consistent with tilt contrast. They displayed no systematic errors in dot localization when instructed to use the axis strategy, demonstrating that tilt contrast depends on the use of the origin strategy. Angle error was a flat function of stimulus angle in the axis strategy condition, with errors centered around zero. The subjects displayed no bias in estimation of origin-to-dot distances. As discussed earlier, these patterns of angle and distance errors can be produced only when subjects do not systematically overor underestimate the positions of stimulus dots along the horizontal and vertical axes (see Figure 3).

\section{GENERAL DISCUSSION}

In three experiments, we investigated the effect of orthogonal axes and axes of symmetry on reproduction of dot location. The subjects reproduced a dot within the frame of reference created by a set of horizontal and vertical axes, either from memory or while viewing the figure. In Experiment 1, memory for location of dots was biased away from the horizontal and vertical axes and the imaginary $45^{\circ}$ line. No systematic pattern of error was observed in the dot-to-origin distance, and it does not appear that the repulsion away from the axes can be explained in terms of bias in memory for the dots' horizontal and vertical positions. The subjects' responses did not display a consistent tilt contrast effect in Experiment 2 (perception condition).

The cyclical pattern of errors observed in memory is consistent with the pattern of distortion found for line reproduction (Schiano \& Tversky, 1992). This suggests that people spontaneously employ the origin strategy, coding the orientation and length of a virtual line from the axes' origin to the dot. Virtual lines are distorted by the same perceptual symmetry extraction mechanisms as real lines (Hartley, 1982), which causes subjects to reproduce dots at an angle tilted away from the nearest axis of symmetry. This interpretation is supported by the results of Experiment 3, in which the subjects were explicitly instructed to reproduce dots by using either the origin strategy or the axis strategy in a perception condition. Tilt contrast was observed only when they used the origin strategy. No systematic angle or distance errors were found when the subjects used the axis strategy.

One major conclusion from the three experiments is that people employ different cognitive strategies to encode spatial location. This is especially clear from the results of Experiment 3, in which the subjects had no difficulty in shifting from the use of the origin strategy to the axis strategy when instructed to do so. This point has been demonstrated in other studies as well. For example, Bryant and Subbiah (in press) had subjects reproduce from memory, or during perception, the location of a dot in a square figure that had distance markers along two sides. In both cases, the subjects were more accurate in reproducing dots located on an intersection of two distance markers than dots located off an intersection, and they 
systematically reproduced off-point dots closer to the intersection of markers than they really were. These findings are consistent with the notion that the subjects established subjective landmarks at the intersection points and used them to code the location of dots in perception and memory. How did they do this? It was found in a fourth experiment that dot relocation was biased toward a subjective intersection only when subjects used a projection strategy. In this strategy, the viewer mentally projects from distance markers on two sides of a square to form an imaginary intersection point, then codes the stimulus dot relative to that subjective landmark. When subjects were instructed to use a different strategy that did not confer landmark status on the imaginary intersection points, there was no attraction of stimulus dots toward them.

Findings such as these indicate that encoding of location depends on the cognitive strategy used at the time of encoding. The display features that serve as reference points are not fixed, but are actively selected by the viewer who brings to bear conceptual knowledge and encoding algorithms or heuristics to process spatial relations in the figure (see also Bryant \& Subbiah, in press; Tversky \& Schiano, 1989). The strategy also determines what spatial variables are important in encoding a dot's position. In the origin strategy, the viewers coded the orientation and distance of virtual lines, or the polar coordinates of the stimulus dots. In the axis strategy, the viewers coded lengths along the horizontal and vertical axes, or the Cartesian coordinates of stimulus dots.

Previous research (e.g., Schiano \& Tversky, 1992; Tversky \& Schiano, 1989) has tended to investigate how the selection of a particular frame of reference biases memory for spatial location. The present experiments extend these findings by demonstrating that different strategies for using a given frame of reference also affect encoding of spatial location. Both the origin and axis strategies make reference to the orthogonal axes, but differ in how those features are used to encode location. The origin encodes the orientation of a virtual line relative to the axes, whereas the axis strategy encodes positions along the two axes. It is possible that subjects explicitly refer to the diagonal in the origin strategy, judging the orientation of the virtual origin-to-dot line with respect to the $45^{\circ}$ line. One argument against this possibility is that Schiano and Tversky found that the diagonal attracted lines rather than repulsed them when subjects were instructed to use the $45^{\circ}$ line as a landmark.

The encoding strategy is clearly important in predicting the kinds of errors the viewer will make in encoding spatial location. In the case of the origin strategy, however, the perceptual mechanisms involved in producing tilt contrast also play a crucial role. Thus, the current experiments also demonstrate that strategic and perceptual factors can combine to produce distortion during encoding of location. The strategy determines what information is coded (e.g., orientation and distance in the origin strategy vs. Cartesian coordinates in the axis strategy) and hence how that information must be obtained. In the pro- cess, the strategy determines what kinds of perceptual mechanisms will affect encoding. The origin strategy leads to a tilt contrast effect because subjects code the orientation of a virtual line from the origin to the dot. The subjects' perception of the virtual line's orientation is then biased by symmetry extraction mechanisms (Beh et al., 1971; Wenderoth et al., 1989). The axis strategy does not require any judgment of orientation and so does not involve the symmetry perception mechanisms that produce tilt contrast. Thus, the origin and axis strategies produce different patterns of error because they activate different perceptual mechanisms and biases during encoding. Therefore, it is important to consider what spatial features of a figure the viewer encodes with his or her strategy to predict what kind of distortion will occur in spatial localization.

A number of questions remain about how people use spatial encoding strategies. First, what other strategies might be used to encode location in the frame of reference used in these studies? Our selection of the origin and axis strategies was not exhaustive, and people might develop other strategies, especially if the constraints of our experimental task were lifted. Also, there is the question of what strategies exist for dealing with other frames of reference, such as those used in previous studies. Presumably, each frame of reference supports a number of unique strategies by virtue of their distinct structural and conceptual features. Take, for example, the use of polar coordinate graphs. Polar graphs employ, like Cartesian graphs, two orthogonal axes. Unlike the case of Cartesian graphs, data in a polar graph are plotted by orientation relative to the axes and radial distance from the origin of the axes. Such graphs typically mark off radial distances with a series of concentric circles. How might a viewer code the location of a dot in this kind of figure? On the one hand, the origin strategy makes use of polar coordinate information, encoding the orientation of a virtual line relative to an axis and the dot's radial distance from the origin. A polar graph might provide even stronger impetus to use this strategy. On the other hand, a polar graph would contain circular distance markers that could also be used to judge position. Research has shown that dot relocation is biased by enclosing figures such as circles (e.g., Nelson \& Chaiklin, 1980), and radial distance markers could introduce bias in dot reproduction. This raises the question of how people decide which strategy to use for a particular frame of reference. What factors determine the choice of strategy, given that more than one exists? The answers to these questions must also depend on the particular frame of reference and should be a function of conceptual factors as well. Thus, whether a figure is interpreted as a graph, map, or abstract symbol should play an important role in determining how it is encoded.

\section{REFERENCES}

Beh, H. C., Wenderoth, P. M., \& Purcell, A. T. (1971). The angular function of a rod-and-frame illusion. Perception \& Psychophysics, 9(4), 353-355. 
Blakemore, C., Carpenter, R. H. S., \& Georgeson, M. A. (1970). Lateral inhibition between orientation detectors in the human visual system. Nature, 228, 37-39.

Bouma, H., \& ANDriessen, J. J. (1970). Induced changes in the perceived orientation of line segments. Vision Research, 10, 333-349.

Bransford, J. D., \& Johnson, M. K. (1972). Contextual prerequisites for understanding: Some investigations of comprehension and recall. Journal of Verbal Learning \& Verbal Behavior, 11, 717-726.

Bryant, D. J., \& SubBiah, I. (in press). Subjective landmarks in perception and memory for spatial location. Canadian Joumal of Experimental Psychology.

CARPENTER, R. H. S., \& BLAKEMORE, C. (1973). Interactions between orientations in human vision. Experimental Brain Research, 18, 287-303.

Cermak, L. S., \& Craik, F. I. M. (Eds.) (1979). Levels of processing in human memory. Hillsdale, NJ: Erlbaum.

Cooley, J. W., Lewis, P. A. W., \& Welch, P. D. (1977). The Fast Fourier Transform and its application to time series analysis. In K. Enslein, A. Ralston, \& H. S. Wilf (Eds.), Statistical methods for digital computers (pp. 377-423). New York: Wiley.

Coren, S., \& Girgus, J. S. (1980). Principles of perceptual organization and spatial distortion: The Gestalt illusions. Journal of Experimental Psychology: Human Perception \& Performance, 6, 404-412.

Hartley, A. A. (1978). The major axis effect: Axes of bilateral symmetry or loci of neural interactions? Perception \& Psychophysics, 23 , 537-541.

HARTLEY, A. A. (1982). The role of axes of symmetry in orientation illusions. Perception \& Psychophysics, 31, 367-375.

Huttenlocher, J., Hedges, L. V., \& Duncan, S. (1991). Categories and particulars: Prototype effects in estimating spatial location. Psychological Review, 98, 352-376.

Lederman, S. J., \& TAYlor, M. M. (1969). Perception of interpolated position and orientation by vision and active touch. Perception \& Psychophysics, 6, 153-159.

Nelson, T. O., \& ChaIKLIN, S. (1980). Immediate memory for spatial location. Journal of Experimental Psychology: Human Learning \& Memory, 6, 529-545.

Rosch, E. (1975). Cognitive reference points. Cognitive Psychology, $7,532-547$.

Russell, R. J. H. (1985). Significance tables for the results of Fast Fourier Transforms. British Journal of Mathematical and Statistical Psychology, 38, 116-119.

Sadalla, E. K., Burroughs, W. J., \& Staplin, L. J. (1980). Reference points in spatial cognition. Journal of Experimental Psychology. Human Learning \& Memory, 6, 516-528.

SChIANO, D. J., \& Tversky, B. (1992). Structure and strategy in encoding simplified graphs. Memory \& Cognition, 20, 12-20.
Tversky, B., \& Schiano, D. J. (1989). Perceptual and conceptual factors in distortions in memory for graphs and maps. Joumal of Experimental Psychology: General, 118, 387-398.

WeNDEROTH, P. (1983). Identical stimuli are judged differently in the orientation and position domains. Perception \& Psychophysics, 33, 399-402.

Wenderoth, P., \& BeH, H. (1977). Component analysis of orientation illusions. Perception, 6, 57-75.

WENDEROTH, P., \& CURTHOYs, I. (1974). On the non-additivity of visual tilt illusions. Quarterly Journal of Experimental Psychology, 26, 549-555.

Wenderoth, P., \& Johnstone, S. (1987). Possible neural substrates for orientation analysis and perception. Perception, 16, 693-709.

Wenderoth, P., Johnstone, S., \& VAN DER ZWAN, R. (1989). Twodimensional tilt illusions induced by orthogonal plaid patterns: Effects of plaid motion, orientation, spatial separation, and spatial frequency. Perception, 18, 25-38

Wenderoth, P., Parkinson, A., \& White, D. (1979). A comparison of visual tilt illusions measured by the techniques of vertical setting, parallel matching, and dot alignment. Perception, 8, 47-57.

\section{NOTES}

1. Using the axis strategy, the subjects could produce the cyclical pattern of positive and negative angle errors predicted by the origin strategy only if they exhibit greater overestimation along the vertical than horizontal at stimulus angles of $5^{\circ}-25^{\circ}$, greater overestimation along the vertical than horizontal at stimulus angles of $30^{\circ}-45^{\circ}$, greater overestimation along the horizontal than vertical at stimulus angles of $45^{\circ}-65^{\circ}$, and greater overestimation along the vertical than horizontal at stimulus angles of $65^{\circ}-85^{\circ}$.

2. Phase was not subjected to analysis. Although it is possible that the subjects' data contained the same periodicities but at different phases (hence different error patterns), their averaged pattern would be a straight line as the different phases canceled one another.

3. Russell (1985) does not provide significance levels for frequency components greater than the sixth for data sets of 32 items because it is difficult to distinguish higher level harmonics from noise. Because each subject's data set was only 17 items in length, the model has a weaker but significant periodicity at the fifth frequency component in addition to the fourth (because 4 does not divide evenly into 17). Thus, the fifth component in the subject's data is not informative and was not analyzed.

(Manuscript received September 16, 1992; revision accepted for publication April 12, 1993.) 